Special Issue Inequality Problems in Contact Mechanics

Prepared using sagej.cls 


\title{
Approximation of a simply supported plate with obstacle
}

\author{
Journal Title \\ $\mathrm{XX}(\mathrm{X}): 2-17$ \\ (C) The Author(s) 0000 \\ Reprints and permission: \\ sagepub.co.uk/journalsPermissions.nav \\ DOI: $10.1177 /$ ToBeAssigned \\ www.sagepub.com/

\section{@SAGE}

\section{Cornel Marius Murea ${ }^{1}$ and Dan Tiba ${ }^{2}$}

\begin{abstract}
We discuss an algorithm for the solution of variational inequalities associated to simply supported plates in contact with a rigid obstacle. Our approach has a fixed domain character, uses just linear equations and approximates both the solution and the corresponding coincidence set. Numerical examples are also provided.
\end{abstract}

\section{Keywords}

free boundary problems, penalization, linear approach

\section{Introduction}

Variational inequalities model many contact problems in elasticity, in fluidsolid interaction problems, change of phase phenomena, other applications from physics, Duvaut and Lions (1976), Kikuchi and Oden (1988), Sofonea and Matei (2009).

There is a rich literature devoted to their theoretical study Elliott and Ockendon (1982), Rodrigues (1987), Kinderlehrer and Stampacchia (2000), or their numerical approximation Glowinski, Lions and Trémolières (1981), Fortin and Glowinski (1983). Let us also mention recent extensions of the theory Sofonea, Migorski and Ochal (2012) and the study of the associated optimization problems, in the setting of the optimal control theory Barbu (1984), Tiba (1990), Neittaanmaki and Tiba (1994) or in shape optimization Neittaanmaki, Sprekels and Tiba (2006).

\footnotetext{
${ }^{1}$ Laboratoire de Mathématiques, Informatique et Applications, Université de Haute Alsace, France ${ }^{2}$ Institute of Mathematics of the Romanian Academy, Bucharest, Romania and Academy of Romanian Scientists, Bucharest, Romania, Email: dan.tiba@imar.ro

Corresponding author:

Cornel Marius Murea, Laboratoire de Mathématiques, Informatique et Applications, Université de Haute Alsace, 6, rue des Frères Lumière, 68093 Mulhouse, France

Email: cornel.murea@uha.fr
} 
The difficulty of such problems is related to the presence of a geometric unknown, the so-called free boundary that has to be found together with the state of the system. It may be a surface or a whole region (called mushy region). Equivalently, in obstacle problems, one may look for the coincidence set (where the solution coincides with the obstacle). Its boundary is the free boundary.

Taking into account such aspects and the usual variational approach to variational inequalities in the PDE's setting, it turns out that methods used in shape optimization may be very useful. We refer to fixed domain methods Neittaanmaki and Tiba (2012) that "hide" the geometric unknowns via various procedures. This idea appears already in Neittaanmaki, Pennanen and Tiba (2009), the novelty here is that we propose an approach using just linear differential equations. That is, we avoid the use of nonlinear equations that appear in the penalization methods in semi-group theory Barbu (1984).

In the setting of variational inequalities and free boundary problems, applications of such ideas have been recently discussed in Halanay, Murea and Tiba (2013), (2016), Murea and Tiba (2016) with reference to elliptic or parabolic obstacle problems, one or two phase Stefan problems, fluid-solid contact models. As examples of applications that may be handled via the fixed domain methods, we mention as well the electrochemical machining process Neittaanmaki, Sprekels and Tiba (2006) or various contact problems related to the deformation of the elastic membranes with obstacle, melting or solidification processes. Applications to financial models (the two-asset American options) are also investigated in Murea and Tiba (2016).

This article is devoted to problems in the elasticity theory of plates, in contact with a rigid body. In the next section, we describe the problem and the new algorithm. The stability of our approach is investigated in Section 3. The last part of the paper is devoted to some relevant numerical experiments. It turns out that our method is very rapid and accurate.

\section{Problem formulation and algorithm}

We start with the well-known model of a simply supported plate, to which supplementary unilateral conditions will be added in the sequel:

$$
\begin{aligned}
\Delta\left(u^{3} \Delta y\right) & =f \text { in } \Omega, \\
y=\Delta y & =0 \text { on } \partial \Omega
\end{aligned}
$$

where $y \in H^{2}(\Omega) \cap H_{0}^{1}(\Omega)$ is the unknown vertical displacement of the plate, $f \in L^{2}(\Omega)$ is the load, $\Omega$ is a sufficiently smooth bounded domain in $\mathbb{R}^{d}, d \leq 3$ and $u \in L^{\infty}(\Omega), u(x) \geq m>0$ a.e. in $\Omega$ is the thickness of the plate. Since $u \in L^{\infty}(\Omega)$, it is known that $y \in W^{2, p}(\Omega)$, with some $p>2$ depending on the dimension, Grisvard (1985), by the Sobolev theorem and regularity results for second order elliptic equations. In the last section, polygonal domains will be also considered. 
The boundary value problem (2.1)-(2.2) is equivalent with the minimization of the energy

$$
\arg \min _{z}\left\{\frac{1}{2} \int_{\Omega} u^{3}(\Delta z)^{2} d x-\int_{\Omega} f z d x\right\}
$$

on the space $H^{2}(\Omega) \cap H_{0}^{1}(\Omega)$, which gives the definition of the weak solution for $(2.1)-(2.2)$.

We introduce a convex closed subset representing the space of admissible displacements:

$$
\mathcal{K}=\left\{z \in H^{2}(\Omega) \cap H_{0}^{1}(\Omega) ; z \geq \psi \text { a.e. in } \Omega\right\}
$$

where $\psi \in H^{2}(\Omega)$ is the obstacle bounding the displacement of the plate and we assume

$$
\psi \leq 0 \text { on } \partial \Omega
$$

Relation (2.5) ensures that $\mathcal{K}$ defined in (2.4) is non-void. In the works Glowinski, Lions and Trémolières (1981), Tiba (2016) other examples of closed convex sets

$$
\begin{gathered}
\widetilde{\mathcal{K}}=\left\{z \in H^{2}(\Omega) \cap H_{0}^{1}(\Omega) ; a \leq \Delta z \leq b \text { a.e. in } \Omega\right\} \\
\widehat{\mathcal{K}}=\left\{z \in H^{2}(\Omega) \cap H_{0}^{1}(\Omega) ; \int_{\Omega} f z d x \geq-1\right\}
\end{gathered}
$$

are discussed in a different context, $a, b \in \mathbb{R}$. We shall investigate the application of our linear numerical approach to clamped plates and such examples (2.6), (2.7), in a subsequent paper.

If the minimization in (2.3) is performed on $\mathcal{K}$, classical arguments give the existence of a unique solution. This is due to the assumed strict positivity of $u \in L^{\infty}(\Omega)$ and to the fact that the first integral in (2.3) defines an equivalent norm on $H^{2}(\Omega) \cap H_{0}^{1}(\Omega)$. Then, the functional (2.3) is coercive and strictly convex.

The above problem can be rewritten as a variational inequality: find $y \in \mathcal{K}$ such that

$$
\int_{\Omega} u^{3} \Delta y(\Delta y-\Delta z) d x \leq \int_{\Omega} f(y-z) d x, \quad \forall z \in \mathcal{K}
$$

It is also possible to re-express $(2.8)$ or $(2.3),(2.4)$ by using maximal monotone operators:

$$
\Delta\left(u^{3} \Delta y\right)+\beta(y-\psi) \ni f \text { in } \Omega,
$$

Prepared using sagej.cls 
together with the boundary conditions (2.2). In fact, (2.8) is a weak formulation of (2.9), where $\beta: \mathbb{R} \rightarrow 2^{\mathbb{R}}$ is the multi-valued function:

$$
\beta(r)=\left\{\begin{array}{cc}
0, & r>0 \\
]-\infty, 0], & r=0 \\
\emptyset, & r<0
\end{array}\right.
$$

and it will not be distinguished from its maximal monotone graph viewed as a subset of $\mathbb{R} \times \mathbb{R}$. The nonlinear term in $(2.9), \beta(y-\psi)$ represents the unknown reaction of the obstacle, when the contact occurs. Classical approximation methods for (2.9), (2.10) use the Yoshida-Moreau penalization:

$$
\beta_{\epsilon}(r)= \begin{cases}\frac{1}{\epsilon} r, & r \leq 0 \\ 0, & r>0\end{cases}
$$

and smoothing procedures with standard mollifiers. This ensures differentiability in the approximating equations, Barbu (1976), Barbu (1984).

We state now our algorithm, that uses just linear equations:

Algorithm 1 (for (2.9), (2.2))

1) Choose $n=0, \epsilon_{0}>0, C_{0} \subset \Omega$ closed (the initial guess of the coincidence set), $y_{-1} \in L^{2}(\Omega)$;

2) Compute $y_{n} \in H^{2}(\Omega)$ and satisfying (2.2) such that

$$
\Delta\left(u^{3} \Delta y_{n}\right)+\frac{1}{\epsilon_{n}} \chi_{C_{n}}\left(y_{n}-\psi\right)=f \text { in } \Omega .
$$

3) $\bar{y}_{n}=\max \left\{y_{n}, \psi\right\}, C_{n+1}=\left\{x \in \Omega ; \bar{y}_{n}(x)=\psi(x)\right\}, \epsilon_{n+1}=\frac{\epsilon_{n}}{2}$;

4) If $\left\|\bar{y}_{n}-\bar{y}_{n-1}\right\|_{L^{2}(\Omega)}<$ tol then STOP; else $n:=n+1$ GO TO step 2.

Notice that $y_{n}$ computed in Step 2 is at least in $H^{2}(\Omega)$ and continuous since $\operatorname{dim}(\Omega) \leq 3$ and the approximating coincidence set $C_{n}$ defined in Step 3 is closed. Here, $\chi_{C_{n}}$ is the characteristic function of $C_{n}$. If we make the convention that in $(2.11)$ :

$$
\beta_{\epsilon}^{\prime}(0)=\frac{1}{\epsilon}
$$

then we get

$$
\frac{1}{\epsilon_{n}} \chi_{C_{n}}=\beta_{\epsilon_{n}}^{\prime}\left(y_{n-1}-\psi\right)
$$

and the equation in Step 2 can be rewritten as

$$
\Delta\left(u^{3} \Delta y_{n}\right)+\beta_{\epsilon_{n}}^{\prime}\left(y_{n-1}-\psi\right)\left(y_{n}-\psi\right)=f \text { in } \Omega,
$$

together with the boundary condition (2.2). This shows the "decoupling" operation (2.14), (2.15) that we perform in the above algorithm. Recalling that, under the convention (2.13), we have $\beta_{\epsilon_{n}}(r)=\beta_{\epsilon}^{\prime}(r) r$, one can compare our linear approach with the classical regularization approach for variational inequalities, Barbu (1984). 


\section{Stability}

We start with two technical lemmas that show that the variational inequality (2.8) associated to the convex (2.4) (see the equivalent formulation (2.9)) can be reduced to the case when the obstacle $\psi$ has zero trace on $\partial \Omega$.

Lemma 3.1. Denote by $\widehat{y} \in H^{2}(\Omega)$ satisfying (2.2), the unique solution of the partial differential equation

$$
\Delta\left(u^{3} \Delta \widehat{y}\right)=f \text { in } \Omega .
$$

Then $y \in \mathcal{K}$, the solution of (2.9), (2.2) satisfies $y(x) \geq \widehat{y}(x)$ a.e. in $\Omega$.

Proof. We know that $\beta(y-\psi) \in L^{2}(\Omega)$ and $\beta(y-\psi) \leq 0$ a.e. in $\Omega$. Denote by $z$, respectively $\widehat{z}$ the solutions of

$$
\begin{aligned}
\Delta z+\beta(y-\psi) & =f \text { in } \Omega, \quad z \in H^{2}(\Omega) \cap H_{0}^{1}(\Omega), \\
\Delta \widehat{z} & =f \text { in } \Omega, \quad \widehat{z} \in H^{2}(\Omega) \cap H_{0}^{1}(\Omega) .
\end{aligned}
$$

Then, by comparison properties for elliptic equations, we have $z \leq \widehat{z}$ a.e. in $\Omega$. We notice that $\Delta y=u^{-3} z, \Delta \widehat{y}=u^{-3} \widehat{z}$ in $\Omega$. Again, by comparison property, we get the conclusion $y \geq \widehat{y}$ a.e. in $\Omega$ since $u$ is strictly positive.

Remark. Assume that $\beta(y-\psi) \neq 0$ in $\Omega$, i.e. $y$ and $\widehat{y}$ cannot satisfy $y=\widehat{y}$ in $\Omega$. We also have $z, \widehat{z} \in H^{2}(\Omega) \cap H_{0}^{1}(\Omega)$ by $(3.2),(3.3)$ and, consequently, $z, \widehat{z} \in$ $W^{1, p}(\Omega), p<6$, by the Sobolev embedding theorem, since $\operatorname{dim}(\Omega) \leq 3$. Under the hypothesis $u \in W^{1, \infty}(\Omega)$, since $u(x) \geq m>0$, we get $u^{-3} z \in W^{1, p}(\Omega), p<$ 6 . Similarly for $\widehat{z}$. It yields that $y, \widehat{y} \in W^{3, p}(\Omega)$ by regularity theory for elliptic equations, Grisvard (1985). Again, the Sobolev theorem gives $y, \widehat{y} \in \mathcal{C}^{2}(\bar{\Omega})$ if $p>3$, which is valid in our case. It turns out that one can use the strong maximum principle Evans (2010), in this setting ( $\partial \Omega$ is sufficiently smooth) and we get the sharper inequality

$$
y(x)>\widehat{y}(x) \text { in } \Omega .
$$

Denote $\widehat{\psi}=\max (\psi, \widehat{y})$ in $\Omega$. Then $\widehat{\psi} \in H_{0}^{1}(\Omega)$ if $\psi \in H^{1}(\Omega)$ and $\psi \leq 0$ on $\partial \Omega$ as assumed. We also have $y \geq \widehat{\psi}$ a.e. in $\Omega$.

Lemma 3.2. The solution $y \in \mathcal{K}$ of (2.9), (2.2) satisfies the same problem with $\psi$ replaced by $\widehat{\psi}$.

Proof. We check some equalities and inequalities: $y>\widehat{\psi} \Rightarrow y>\psi \Rightarrow \beta(y-$ $\widehat{\psi})=\beta(y-\psi)=0 ; y=\widehat{\psi}=\max (\psi, \widehat{y}) \Rightarrow \widehat{\psi}=\psi \Rightarrow y=\psi$ since $y>\widehat{y}$ by (3.4). It yields that $\beta(y-\widehat{\psi})=\beta(y-\psi)$ in $\Omega$ and $(2.9)$ is valid with $\psi$ replaced by $\widehat{\psi}$. Since $y \geq \widehat{\psi}$ a.e. in $\Omega$, we can replace $\psi$ by $\widehat{\psi}$ in the definition of $\mathcal{K}$ as well. Relation (2.2) remains valid. This ends the proof.

Due to the above results, we can make the assumption $\psi \in H_{0}^{1}(\Omega)$ and prove 
Theorem 3.1. If $\partial \Omega$ is of class $\mathcal{C}^{1,1}, \psi \in H^{2}(\Omega) \cap H_{0}^{1}(\Omega), u \in W^{1, \infty}(\Omega)$, $u(x) \geq m>0$ a.e. in $\Omega$, then we have:

i) On a subsequence, $y_{n} \rightarrow \widetilde{y}$ weakly in $H^{2}(\Omega) \cap H_{0}^{1}(\Omega)$ and $\Omega \backslash C_{n} \rightarrow \Omega \backslash C$ in the Hausdorff-Pompeiu complementary topology.

ii) If $\Omega \backslash C_{n}$ are uniformly of class $\mathcal{C}$ (see Neittaanmaki, Sprekels and Tiba (2006)) and the convergence is valid on the whole sequence, then $C$ is the coincidence set corresponding to (2.9), (2.2) and $\widetilde{y}$ satisfies (2.9), (2.2) in a weak sense explained below.

Proof. i) We multiply (2.12) or (2.15) by $y_{n}-\psi \in H_{0}^{1}(\Omega)$ and integrate by parts:

$$
\int_{\Omega} u^{3} \Delta y_{n} \Delta\left(y_{n}-\psi\right) d x+\frac{1}{\epsilon_{n}} \int_{C_{n}}\left(y_{n}-\psi\right)^{2} d x=\int_{\Omega} f\left(y_{n}-\psi\right) d x .
$$

The positivity of the second integral in (3.5) gives:

$$
\int_{\Omega} u^{3}\left|\Delta y_{n}\right|^{2} d x \leq \int_{\Omega} u^{3} \Delta y_{n} \Delta \psi d x+\int_{\Omega} f\left(y_{n}-\psi\right) d x .
$$

It yields that $\left\{y_{n}\right\}$ is bounded in $H^{2}(\Omega)$ since the first integral in (3.6) defines an equivalent norm on $H^{2}(\Omega)$ due to the zero boundary conditions. We also obtain:

$$
\int_{C_{n}}\left(y_{n}-\psi\right)^{2} d x \leq c \epsilon_{n}
$$

with $c>0$ independent of $n \in \mathbb{N}$. We denote by $\widetilde{y} \in H^{2}(\Omega) \cap H_{0}^{1}(\Omega)$ the weak limit in $H^{2}(\Omega)$ of $y_{n}$ and $C=\lim C_{n}$ in the Hausdorff-Pompeiu topology for compact sets in Euclidean spaces, on a subsequence.

ii) Denote $D=\Omega \backslash C$ open subset and let $\rho \in \mathcal{C}_{0}^{\infty}(D)$. By the $\Gamma$ property for the complementary Hausdorff-Pompeiu convergence, Neittaanmaki, Sprekels and Tiba (2006), Appendix 3, we get $\rho \in \mathcal{C}_{0}^{\infty}\left(\Omega \backslash C_{n}\right)$, for $n$ sufficiently big. We multiply (2.12) by $\rho$ and obtain

$$
\int_{\Omega} u^{3} \Delta y_{n} \Delta \rho d x=\int_{\Omega} f \rho d x
$$

after partial integration and since $C_{n} \cap \operatorname{supp}(\rho)=\emptyset$. One can pass to the limit in (3.8) and obtain

$$
\int_{\Omega} u^{3} \Delta \tilde{y} \Delta \rho d x=\int_{\Omega} f \rho d x, \quad \forall \rho \in \mathcal{C}_{0}^{\infty}(D)
$$

that is $\widetilde{y}$ satisfies in $D$ the equation (2.1), in the sense of distributions.

Under the supplementary assumption that $\Omega \backslash C_{n}$ are domains of class $\mathcal{C}$ (see Neittaanmaki, Sprekels and Tiba (2006), Appendix 3) we also get the convergence of the corresponding characteristic functions, a.e. in $\Omega$. By 
inequality (3.7), we obtain

$$
\int_{C}(\widetilde{y}-\psi)^{2} d x=0
$$

that is $\widetilde{y}(x)=\psi(x)$ in $C$ (here also use the continuity of $\widetilde{y}, \psi$ since $H^{2}(\Omega) \subset \mathcal{C}(\bar{\Omega})$ for $\operatorname{dim}(\Omega) \leq 3)$. As $D$ is of class $\mathcal{C}$, then $\partial D$ has zero Lebesgue measure and $D \cup C=\Omega$, up to a set of zero measure. Combining this with (3.9) and the conclusion of (3.10), we see that (2.9) is satisfied by $\widetilde{y}$ in a generalized sense. The same is valid for (2.2) in the sense that all $y_{n}$ satisfy it and we have the convergence property. Obviously, $\widetilde{y}$ satisfies (2.4) since $y_{n}>\psi$ outside $C_{n+1}$ by Algorithm 1 and we can again apply the $\Gamma$ property for the sequence $\Omega \backslash C_{n}$. Here, we also use the convergence assumption from ii).

Remark. Notice that the above convergence of $y_{n}$ is also valid in the uniform topology.

\section{Numerical experiments}

We assume that the thickness of the plate is constant $u=1$. Let $V_{h} \subset H^{2}(\Omega) \cap$ $H_{0}^{1}(\Omega)$ be a finite dimensional subspace. Classically, see Dautray and Lions (1990), Chapter XII, section 4, we can approach (2.12) by: find $y_{n, h} \in V_{h}$ such that

$$
\int_{\Omega}\left(\Delta y_{n, h}\right)\left(\Delta z_{h}\right) d x+\frac{1}{\epsilon_{n}} \int_{\Omega} \chi_{C_{n}}\left(y_{n, h}-\psi_{h}\right) z_{h} d x=\int_{\Omega} f z_{h} d x, \quad \forall z_{h} \in V_{h}
$$

where $\psi_{h} \in V_{h}$ is an approximation of $\psi$ and we have assumed that $\psi=0$ on $\partial \Omega$. This discrete problem has a unique solution. As in the continuous case, $\left\|\Delta y_{n, h}\right\|_{L^{2}(\Omega)}$ is bounded and $\left\|y_{n, h}-\psi_{h}\right\|_{L^{2}\left(C_{n}\right)} \leq C \sqrt{\epsilon_{n}}$, where $C>0$ is a constant. But the drawback of this approach is to use high order polynomial finite element approximation to have $V_{h} \subset H^{2}(\Omega)$.

We follow a different strategy, the mixed finite element method, see Boffi, Brezzi and Fortin (2013). With the notation $\omega_{n}=-\Delta y_{n}$, the problem (2.12), (2.2) is equivalent to

$$
\begin{aligned}
-\Delta y_{n} & =\omega_{n}, \quad \text { in } \Omega \\
-\Delta \omega_{n}+\frac{1}{\epsilon_{n}} \chi_{C_{n}}\left(y_{n}-\psi\right) & =f, \quad \text { in } \Omega \\
y_{n} & =0, \quad \text { on } \partial \Omega \\
\omega_{n} & =0, \quad \text { on } \partial \Omega .
\end{aligned}
$$

Let $f \in L^{2}(\Omega)$. Then, the weak solution of (2.12), (2.2) satisfies $y_{n} \in H^{2}(\Omega) \cap$ $H_{0}^{1}(\Omega)$. As $u=1$, the above notation and (2.12) give that $\omega_{n} \in L^{2}(\Omega)$ is the unique transposition solution of the Dirichlet problem for the Laplace equation (the penalization term is "known" in $L^{2}(\Omega)$ ). As $\Omega$ is smooth, elliptic regularity theory gives $\omega_{n} \in H^{2}(\Omega) \cap H_{0}^{1}(\Omega)$ and, using (4.1), it yields $y_{n} \in$ 
$H^{4}(\Omega)$. Multiplying (4.1) by $\mu \in H_{0}^{1}(\Omega)$ and (4.2) by $\phi \in H_{0}^{1}(\Omega)$ and integrating by parts, we obtain the mixed problem: find $y_{n}, \omega_{n} \in H_{0}^{1}(\Omega)$ such that

$$
\begin{aligned}
& \int_{\Omega} \omega_{n} \mu d x-\int_{\Omega} \nabla y_{n} \cdot \nabla \mu d x=0, \quad \forall \mu \in H_{0}^{1}(\Omega) \\
& -\int_{\Omega} \nabla \omega_{n} \cdot \nabla \phi d x-\frac{1}{\epsilon_{n}} \int_{\Omega} \chi_{C_{n}}\left(y_{n}-\psi\right) \phi d x \\
& =-\int_{\Omega} f \phi d x, \quad \forall \phi \in H_{0}^{1}(\Omega) .
\end{aligned}
$$

The minus sign in (4.6) is in order to obtain a symmetric matrix. For the existence, uniqueness and finite element approximation for the biharmonic problem with clamped boundary conditions, we refer to Boffi, Brezzi and Fortin (2013), Chapter 10. If $\Omega$ is a bounded polygonal domain in $\mathbb{R}^{2}$, the solution of the simply supported plate problem is only in $H^{2+\alpha}(\Omega)$, where $\alpha \in(0,1]$ is the index of elliptic regularity determined by the interior angles of $\Omega$, see Brenner et al (2015) and the references therein.

The formulation (4.5), (4.6) allows us to use only $\mathbb{P}_{1}$ triangular finite element for $y_{n}$ as well as for $\omega_{n}$ in all tests. We assume that $\Omega$ is a bounded polygonal domain in $\mathbb{R}^{2}$ and let $\mathcal{T}_{h}$ be a triangulation of $\Omega$. We introduce the finite dimensional space

$$
W_{h}=\left\{w_{h} \in \mathcal{C}(\bar{\Omega}) ; \forall T \in \mathcal{T}_{h}, w_{h \mid T} \in \mathbb{P}_{1}(T)\right\}
$$

and the discrete mixed problem: find $y_{n, h}, \omega_{n, h} \in W_{h}$ such that

$$
\begin{aligned}
& \int_{\Omega} \omega_{n, h} \mu_{h} d x-\int_{\Omega} \nabla y_{n, h} \cdot \nabla \mu_{h} d x=0, \quad \forall \mu_{h} \in W_{h} \\
& -\int_{\Omega} \nabla \omega_{n, h} \cdot \nabla \phi_{h} d x-\frac{1}{\epsilon_{n}} \int_{\Omega} \chi_{C_{n, h}}\left(y_{n, h}-\psi_{h}\right) \phi_{h} d x \\
& =-\int_{\Omega} f \phi_{h} d x, \quad \forall \phi_{h} \in W_{h} .
\end{aligned}
$$

If not otherwise specified, the initial guess of the coincidence set is $C_{0}=\emptyset$. We have used the software FreeFem ++ , Hecht (2012).

Test 1. We have adapted Example 2 from Brenner et al (2012) for the homogeneous simply supported boundary conditions.

The data are: the domain $\Omega=(-0.5,0.5)^{2}$, the load $f=0$, the obstacle $\psi(x)=1-5|x|^{2}+|x|^{4}$ and the boundary conditions $y=0$ and $\Delta y=0$ on $\partial \Omega$.

We use a mesh of 153962 triangles, 77494 vertices and size $h=2^{-8}$. The tolerance for the stopping test in Algorithm 1 is $t o l=10^{-8}$ and the penalization parameter is fixed to $\epsilon_{n}=10^{-5}$. Our algorithm stops after $n=8$ iterations and the errors between the last two iterations are: $\left\|\bar{y}_{n}-\bar{y}_{n-1}\right\|_{L^{2}(\Omega)}=3.6 e-9$, $\left\|\bar{y}_{n}-\bar{y}_{n-1}\right\|_{H^{1}(\Omega)}=1.5 e-7$. The computed coincidence set and solution are presented in Figures 1 and 2.

As underlined in the previous section, we can use Algorithm 1 with $\psi$ replaced by $\widehat{\psi}=\max (\psi, \widehat{y})$ in $\Omega$, where $\widehat{y}$ is the solution of (3.1), (2.2). In this setting, we 


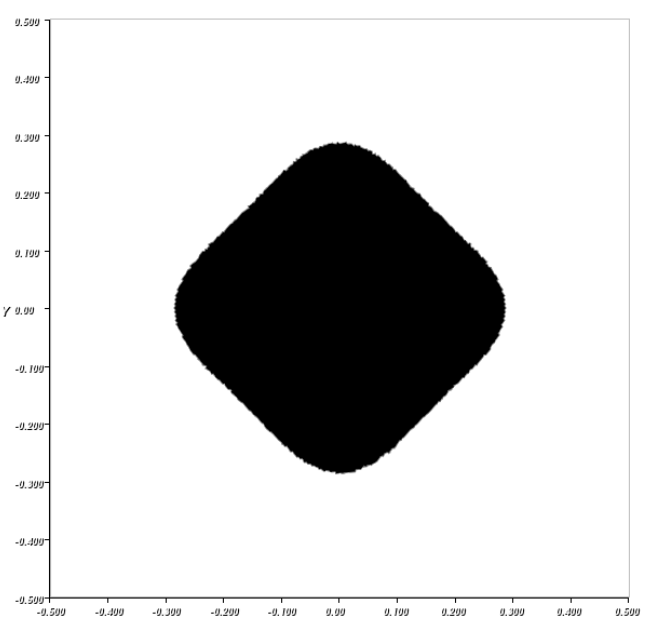

Figure 1. The coincidence set for the Test 1.
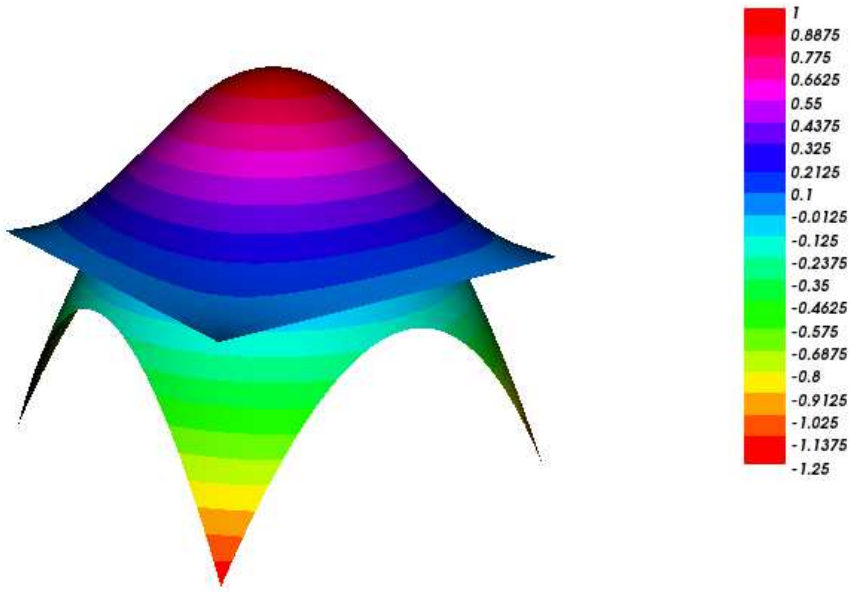

Figure 2. The computed solution with the obstacle for the Test 1.

can start, for instance, with $C_{0}=\Omega$ and the other numerical parameters are as before. Our algorithm stops after $n=7$ iterations and the error between the last two iterations is zero. For $n=6$ the errors are $\left\|\bar{y}_{n}-\bar{y}_{n-1}\right\|_{L^{2}(\Omega)}=1.14 e-6$, $\left\|\bar{y}_{n}-\bar{y}_{n-1}\right\|_{H^{1}(\Omega)}=3.99 e-5$. The error in the norm $L^{\infty}(\Omega)$ between the solutions obtained using $\psi$ and respectively $\widehat{\psi}$ is $2.65 e-6$.

Test 2. It is the one obstacle problem from Brenner et al (2015). 
The data are: L-shaped domain $\Omega=(-0.5,0.5)^{2} \backslash([0,0.5] \times[-0.5,0])$, the load $f=0$, the obstacle $\psi(x)=1-\frac{\left(x_{1}+0.25\right)^{2}}{0.24^{2}}-\frac{\left(x_{2}-0.25\right)^{2}}{0.24^{2}}$ and the boundary conditions $y=0$ and $\Delta y=0$ on $\partial \Omega$.

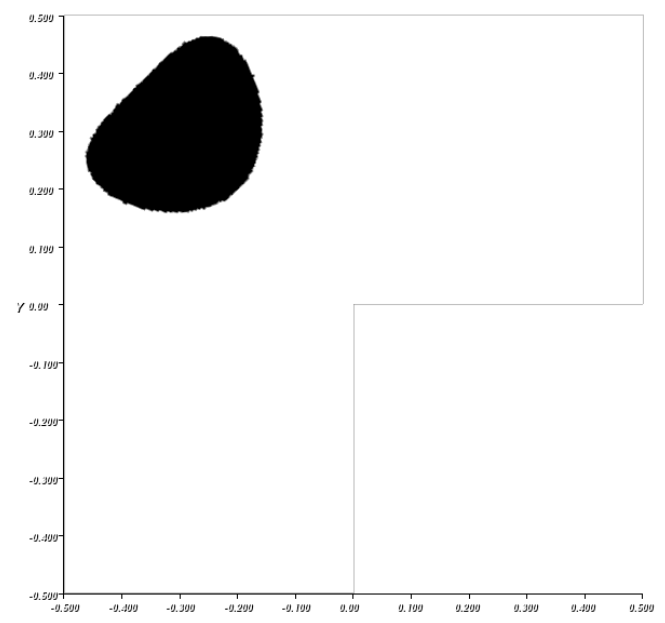

Figure 3. The coincidence set for the Test 2.
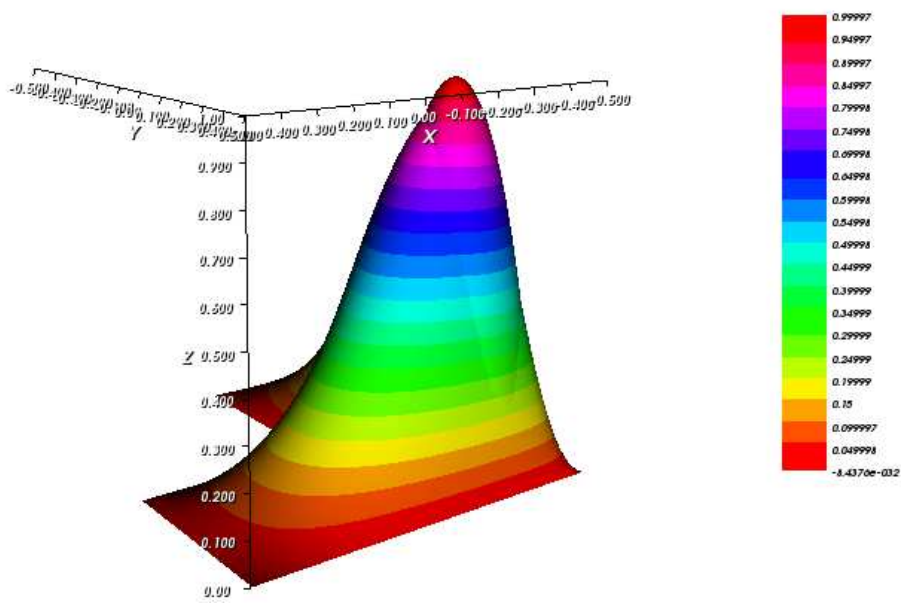

Figure 4. The computed solution for the Test 2.

We use a mesh of 110730 triangles, 55878 vertices and size $h=2^{-7}$. The tolerance for the stopping test in Algorithm 1 is $t o l=10^{-5}$ and the penalization parameter is fixed to $\epsilon_{n}=10^{-5}$. Our algorithm stops after $n=7$ iterations 
and the error between the last two iterations is zero. For $n=6$ the errors are $\left\|\bar{y}_{n}-\bar{y}_{n-1}\right\|_{L^{2}(\Omega)}=3.0 e-5,\left\|\bar{y}_{n}-\bar{y}_{n-1}\right\|_{H^{1}(\Omega)}=5.0 e-4$. We obtain a symmetric coincidence set, slightly larger than in Brenner et al (2015), see Figure 3.

Test 3. We have adapted Example 4 from Brenner et al (2012) for the homogeneous simply supported boundary conditions.

The data are: L-shaped domain from the Test 2 , the load $f=0$, the obstacle $\psi(x)=1-\frac{\left(x_{1}+0.25\right)^{2}}{0.2^{2}}-\frac{x_{2}^{2}}{0.35^{2}}$ and the boundary conditions $y=0$ and $\Delta y=0$ on $\partial \Omega$.

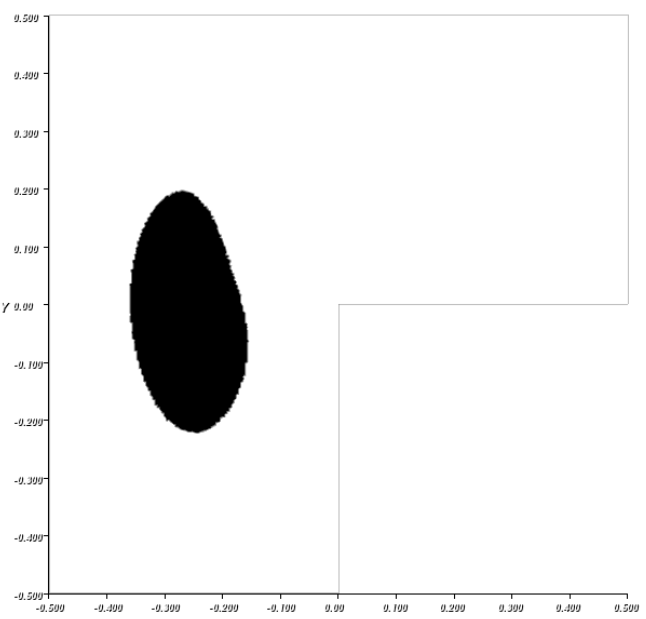

Figure 5. The coincidence set for the Test 3.

We have used the same mesh as in Test 2. The tolerance for the stopping test in Algorithm 1 is $t o l=10^{-5}$ and the penalization parameter is fixed to $\epsilon_{n}=10^{-5}$. Our algorithm stops after $n=7$ iterations and the error between the last two iterations is zero. For $n=6$ the errors are $\left\|\bar{y}_{n}-\bar{y}_{n-1}\right\|_{L^{2}(\Omega)}=2.5 e-6$, $\left\|\bar{y}_{n}-\bar{y}_{n-1}\right\|_{H^{1}(\Omega)}=6.7 e-5$.

Test 4. The algorithm works in more general situations than investigated in Section 3. We have adapted Example 1 from Brenner et al (2012) for the non-homogeneous simply supported boundary conditions.

The data are: the domain $\Omega=(-0.5,0.5)^{2}$, the load $f=0$, the obstacle $\psi(x)=1-|x|^{2}$ where $|x|=\sqrt{x_{1}^{2}+x_{2}^{2}}$ and the boundary conditions $y=y_{e}$ and $\Delta y=\Delta y_{e}$ on $\partial \Omega$, where $y_{e}$ is the exact solution of the obstacle problem for the non-homogeneous simply supported plate given by

$$
y_{e}(x)= \begin{cases}C_{1}|x|^{2} \ln (|x|)+C_{2}|x|^{2}+C_{3} \ln (|x|)+C_{4}, & r_{0}<|x|<2, \\ 1-|x|^{2}, & |x| \leq r_{0}\end{cases}
$$

where $r_{0}=0.18134452, C_{1}=0.52504063, C_{2}=-0.62860904, C_{3}=0.01726640$, $C_{4}=1.04674630$.

Prepared using sagej.cls 

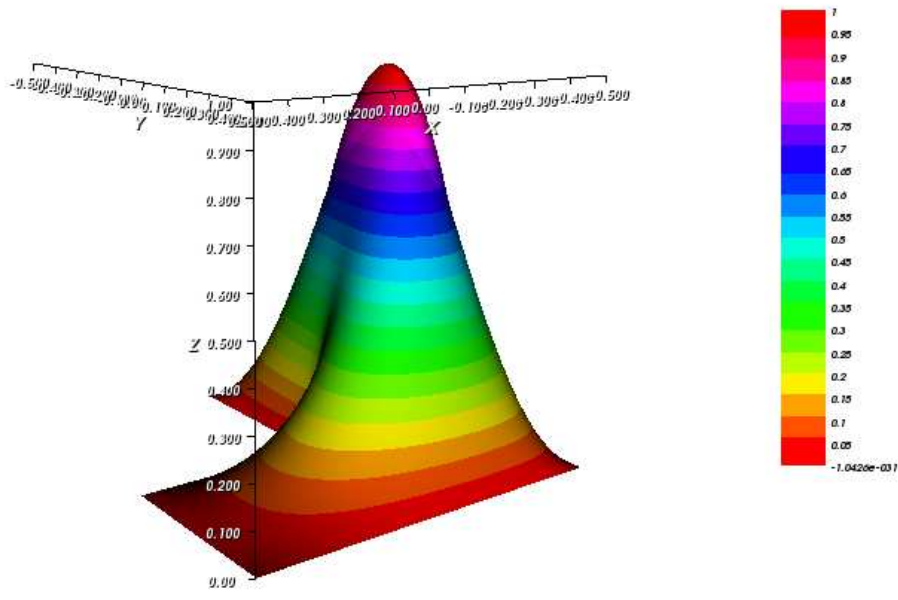

Figure 6. The computed solution for the Test 3.

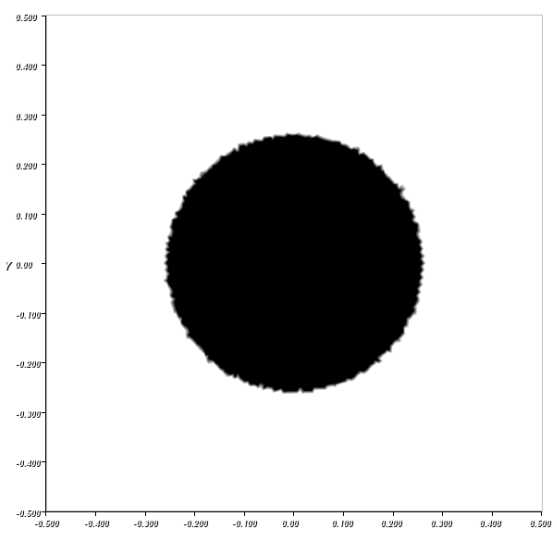

Figure 7. The coincidence set for the Test 4.

We use a mesh of 39034 triangles, 19774 vertices and size $h=2^{-7}$. The tolerance for the stopping test in Algorithm 1 is $t o l=10^{-8}$ and the penalization parameter is fixed to $\epsilon_{n}=10^{-5}$. Our algorithm stops after $n=8$ iterations and the errors between the computed and the exact solutions are: $\left\|\bar{y}_{n}-y_{e}\right\|_{L^{2}(\Omega)}=$ $3.4 e-4,\left\|\bar{y}_{n}-y_{e}\right\|_{H^{1}(\Omega)}=4.8 e-3,\left\|\bar{y}_{n}-y_{e}\right\|_{L^{\infty}(\Omega)}=7.6 e-4$ which prove the algorithm is effective. The history of error of computed solution between two consecutive iterations is presented in Table 1.

The exact coincidence set is the disk $\left\{x \in \Omega ;|x| \leq r_{0}\right\}$. We obtain numerically a slightly larger disk, see Figure 7 . This is a delicate test and it is due to the 

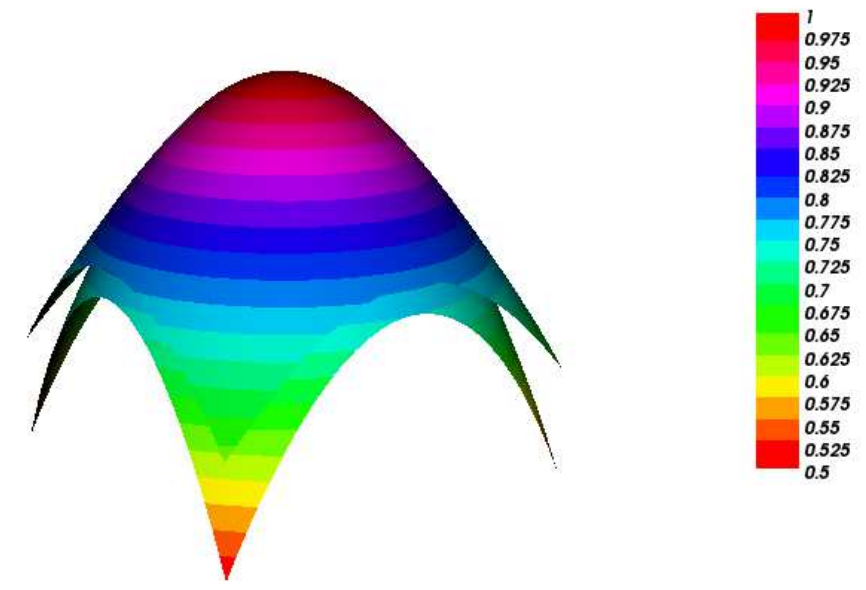

Figure 8. The computed solution with the obstacle for the Test 4.

\begin{tabular}{|c|c|c|c|c|c|}
\hline iteration & 3 & 4 & 5 & 6 & 7 \\
\hline$\left\|\mathbf{y}_{n}-\mathbf{y}_{n-1}\right\|_{L^{2}(D)}$ & 0.002015 & 0.000737 & 0.000143 & $7.11 \mathrm{e}-06$ & $7.26 \mathrm{e}-08$ \\
\hline$\left\|\mathbf{y}_{n}-\mathbf{y}_{n-1}\right\|_{H^{1}(D)}$ & 0.024636 & 0.009580 & 0.002746 & 0.000171 & $1.81 \mathrm{e}-06$ \\
\hline
\end{tabular}

Table 1. History of the errors between two consecutive iterations for the Test 4.

employed precision, because the exact solution is very close to the obstacle in a close neighborhood of the coincidence set, for example $y_{e}(0,0.25)-\psi(0,0.25)=$ 0.00053 , see Figure 9.

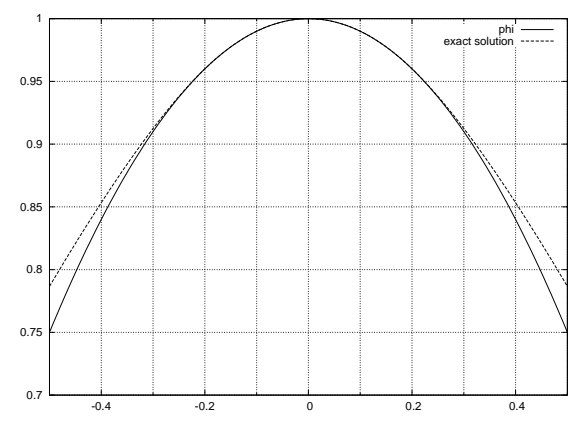

Figure 9. The exact solution and the obstacle for the Test 4. Cut by the plane $x_{1} O x_{3}$ 
In order to estimate the convergence order $q$ (see Theodor (1982)) from the formula

$$
\lim _{n \rightarrow \infty} \frac{\left\|\bar{y}_{n+1}-y_{e}\right\|_{L^{2}(\Omega)}}{\left\|\bar{y}_{n}-y_{e}\right\|_{L^{2}(\Omega)}^{q}}<+\infty,
$$

we have computed the slope of the regression line of the points

$$
\left(\log _{10}\left(\left\|\bar{y}_{n}-y_{e}\right\|_{L^{2}(\Omega)}\right), \log _{10}\left(\left\|\bar{y}_{n+1}-y_{e}\right\|_{L^{2}(\Omega)}\right)\right), n=0, \ldots
$$

and similarly for the norm of $H^{1}(\Omega)$. The results indicate in this example that the convergence order of Algorithm 1 is $q=1.19$ for the norm of $L^{2}(\Omega)$ and $q=1.3$ for the norm of $H^{1}(\Omega)$. We used also initial guess of the coincidence set $C_{0}=\{x \in \Omega ;|x| \leq 0.1\}$ and $C_{0}=\{x \in \Omega ;|x| \leq 0.05\}$ and the results are the same.

\section{Acknowledgements}

The authors thank the anonymous referees for their very helpful reports that improved the quality of the presentation.

\section{References}

Duvaut G and Lions JL (1976) Inequalities in Mechanics and Physics, Berlin, New York: Springer-Verlag.

Kikuchi N and Oden JT (1988) Contact problems in elasticity: a study of variational inequalities and finite element methods. SIAM Studies in Applied Mathematics, 8. Philadelphia: Society for Industrial and Applied Mathematics (SIAM).

Sofonea M and Matei A (2009) Variational Inequalities with Applications. A Study of Antiplane Frictional Contact Problems, Advances in Mechanics and Mathematics, Berlin: Springer.

Elliott CM and Ockendon JR (1982) Weak and Variational Methods for Moving Boundary Problems. Research Notes in Mathematics, 59. London: Pitman.

Rodrigues JF (1987) Obstacle Problems in Mathematical Physics. Amsterdam: NorthHolland Publishing Co..

Kinderlehrer D and Stampacchia G (2000) An Introduction to Variational Inequalities and Their Applications. Reprint of the 1980 original. Classics in Applied Mathematics, 31, Philadelphia: Society for Industrial and Applied Mathematics (SIAM).

Glowinski R, Lions JL and Trémolières R (1981) Numerical Analysis of Variational Inequalities. Studies in Mathematics and its Applications, 8. Amsterdam, New York: North-Holland Publishing Co.

Fortin M and Glowinski R (1983) Augmented Lagrangian methods. Applications to the numerical solution of boundary value problems. Studies in Mathematics and its Applications, 15. Amsterdam: North-Holland Publishing Co.

Sofonea M, Migorski S and Ochal A (2012) Nonlinear inclusions and hemivariational inequalities: models and analysis of contact problems, New York: Springer. 
Barbu V (1984) Optimal Control of Variational Inequalities. Research Notes in Mathematics, 100, Boston: Pitman.

Tiba D (1990) Optimal Control of Nonsmooth Distributed Parameter Systems. Berlin: Springer.

Neittaanmaki P and Tiba D (1994) Optimal Control of Nonlinear Parabolic Systems. Theory, Algorithms, and Applications, Monographs and Textbooks in Pure and Applied Mathematics, 179. New York: Marcel Dekker Inc..

Neittaanmaki P, Sprekels J and Tiba D (2006) Optimization of Elliptic Systems. Theory and Applications. Springer Monographs in Mathematics. New York: Springer.

Neittaanmaki P and Tiba D (2012) Fixed domain approaches in shape optimization problems, Inverse Problems 28: 1-35.

Neittaanmaki P, Pennanen A and Tiba D (2009) Fixed domain approaches in shape optimization problems with Dirichlet boundary conditions, Inverse Problems 25: $1-18$.

Halanay A, Murea CM and Tiba D (2013) Existence and approximation for a steady fluid-structure interaction problem using fictitious domain approach with penalization, Ann. Acad. Rom. Sci. Ser. Math. Appl. 5: 120-147.

Halanay A, Murea CM and Tiba D (2016) Existence of a steady flow of Stokes fluid past a linear elastic structure using fictitious domain, J. Math. Fluid Mech. 18: $397-413$.

Murea CM and Tiba D (2016) A direct algorithm in some free boundary problems. Journal of Numerical Mathematics 24: 253-271.

Tiba D (2016) A duality approximation of some nonlinear PDEs, Ann. Acad. Rom. Sci. Ser. Math. Appl. 8: 68-77.

Barbu V (1976) Nonlinear Semigroups and Differential Equations in Banach Spaces. Leyden: Noordhoff International Publishing.

Grisvard P (1985) Elliptic Problems in Nonsmooth Domains. London: Pitman.

Evans LC (2010) Partial Differential Equations, Providence, Rhode Island: American Mathematical Society.

Dautray R and Lions JL (1990) Mathematical analysis and numerical methods for science and technology. Vol. 4. Integral equations and numerical methods. Berlin: Springer-Verlag.

Boffi D, Brezzi F and Fortin M (2013) Mixed finite element methods and applications. Springer Series in Computational Mathematics, 44. Heidelberg: Springer.

Brenner SC, Gu S, Gudi T and Sung L (2015) A Partition of Unity Method for the Obstacle Problem of Simply Supported Kirchhoff Plates, in Griebel M and Schweitzer MA (eds), Meshfree Methods for Partial Differential Equations VII, Vol. 100 Lecture Notes in Computational Science and Engineering, Springer, pp $23-41$.

Hecht F (2012) New development in FreeFem++. J. Numer. Math. 20: 251-265. http://www.freefem.org

Brenner SC, Sung L, Zhang H and Zhang T (2012) A quadratic $C^{0}$ interior penalty method for the displacement obstacle problem of clamped Kirchhoff plate. SIAM 
J. Numer. Anal. 50: 3329-3350.

Théodor R (1982) Initiation à l'analyse numérique. Paris: Masson. 